\title{
Risk Management in Laser Treatment of Varicose Vein of Lower Extremity with High Ligation of Great Saphenous Vein
}

\author{
Hongtao Xiong1, ${ }^{1,}$, Yongqing Zhuang ${ }^{1}$, Xiaokuan Fu' ${ }^{1}$, Xichi Fang ${ }^{1}$, Zhouyong Tan $^{1}$, Ruihong Wei ${ }^{1}$, Yu- \\ long Sun ${ }^{2}$ \\ ${ }^{1}$ Department of Hand and Microvascular Surgery, Shenzhen People’s Hospital (The Second Clinical Medical College, Jinan Univer- \\ sity; The First Affiliated Hospital, Southern University of Science and Technology), Shenzhen 518020, Guangdong, China. \\ ${ }^{2}$ Shenzhen Key Laboratory of Musculoskeletal Tissue Reconstruction and Function Restoration, (ZDSYS20200811143752005), \\ Shenzhen People's Hospital (The Second Clinical Medical College, Jinan University; The First Affiliated Hospital, Southern Univer- \\ sity of Science and Technology), Shenzhen 518055, Guangdong, China.
}

How to cite this paper: Hongtao Xiong, Yongqing Zhuang, Xiaokuan Fu, Xichi Fang, Zhouyong Tan, Ruihong Wei, Yulong Sun. (2021) Risk Management in Laser Treatment of Varicose Vein of Lower Extremity with High Ligation of Great Saphenous Vein. International Journal of Clinical and Experimental Medicine Research, 5(3), 278-283. DOI: 10.26855/ijcemr.2021.07.008

Received: April 20, 2021

Accepted: May 15, 2021

Published: May 31, 2021

*Corresponding author: Hongtao Xiong, Department of Hand and Microvascular Surgery, Shenzhen People's Hospital (The Second Clinical Medical College, Jinan University; The First Affiliated Hospital, Southern University of Science and Technology), Shenzhen 518020, Guangdong, China.

Email: xionghongtaosz@126.com

\begin{abstract}
Objective: High ligation of great saphenous vein combined with laser in the treatment of varicose veins of lower limbs is a minimally invasive and efficient operation, which is widely used. However, there is less discussion on the risk control and management of this kind of operation. So the study was undertaken to identify the hidden dangers and risks and improve the safety of operation. Methods: 214 cases (201 patients) of lower extremity treated by high ligation of great saphenous vein combined with laser in varicose vein cavity were treated from January 2016 to December 2018. The complications, adverse events and treatment were analyzed. There were no great saphenous vein, root injury of great saphenous vein, guide wire entering deep vein, guide wire or cannula breaking or breaking, skin nerve burn, local skin and soft tissue thermal injury in the groin incision. The patients were followed up for 1 month to 3 years. Results: 30 cases $(14.0 \%)$ had risk or adverse events. During the operation, there were 7 cases without great saphenous vein in the groin incision, 3 cases with root injury of great saphenous vein, 3 cases with guide wire entering deep vein, 1 case with guide wire breaking, and 1 case with cannula burning through and breaking. In the follow-up, 8 cases had local skin and soft tissue thermal injury, 3 cases had saphenous nerve burn, 2 cases had lower extremity deep vein thrombosis and 2 cases had recurrence operated again. Other patients had no adverse consequences. Conclusion: Open operation and intracavitary interventional treatment are better than single operation, but at the same time, they have double risks. During and after the operation, appropriate measures should be taken according to the causes to avoid serious consequences.
\end{abstract}

\section{Keywords}

High Ligation of Great Saphenous Vein, Varicose Vein of Lower Extremity, Intracavitary Laser Therapy, Surgical Risk, Intraoperative and Postoperative Management 


\section{Introduction}

Varicose vein of lower extremities is a common disease in middle-aged and elderly population. The traditional surgical treatment is the high ligation of the great saphenous vein and superficial vein stripping, which leads to the greater trauma. In the past 20 years, endovenous laser treatment (EVLT) has been widely used due to its advantages of minimal invasion and safety [1-2]. Its principle is to use high-energy laser to fuse the vein wall and to achieve the purposes of the vein closure and the treatment [3-4]. The combination of high ligation of great saphenous vein and endovascular laser treatment has a number of advantages [5-6], such as effective and safe, minimally invasive, easy to learn and easy to perform. But the results of operation are associated to the performance of technologies [7-8]. During the operation, many risks and complications could arise, which were summarized in this study.

\section{Subjects \& Methods}

The 214 limbs (201 patients), 117 limbs (110 patients) and 97 limbs (91 patients) were randomly selected from patients, whose varicose veins of the lower extremities were treated by laser in 2016, 2017 and 2018 respectively. The average age was $(57 \pm 22)$ years. The course of disease was 2 to 40 years. All patients were examined by color Doppler ultrasound before operation, to confirm the presence of saphenous femoral valve regurgitation, the deep vein wall was not thick, the diameter was normal, and there was no obvious abnormal echo in the lumen. The diagnosis was consistent with primary varicose vein of lower extremity. Some patients were complicated with hypertension, diabetes, coronary heart disease and so on. Before operation, the condition of other specialties was stable and well controlled.

The preoperative evaluation of varicose veins of the lower extremities showed superficial varicose dilatation, some patients were accompanied with pigmentation, ulcer, etc. according to the CEAP 0-6 level diagnostic criteria of varicose veins of the lower extremities, it was in the range of 3-6 levels. There are 60 lower limbs in grade 3, 92 in grade 4, 38 in Grade 5 and 14 in Grade 6. Routine special physical examination includes visual examination, palpation and deep vein patency test. Auxiliary examination included lower extremity vein color ultrasound, chest radiograph, electrocardiogram, blood routine, coagulation, blood type, blood biochemistry (liver and kidney function, electrolyte, blood sugar), urine routine, blood immunity (hepatitis B, hepatitis C, syphilis and AIDS), exclusion of lower extremity deep vein thrombosis, hemangioma, arteriovenous malformation, etc.

The largest caliber of varicose great saphenous vein is mostly located in upper thigh, lower thigh and upper leg. The diameter of upper thigh is $5 \mathrm{~mm}-15 \mathrm{~mm}$, and that of lower thigh is $4 \mathrm{~mm}-16 \mathrm{~mm}$. The diameter of the upper leg is $5 \mathrm{~mm}-16 \mathrm{~mm}$.

All 214 lower limbs of 201 patients were treated by high ligation of great saphenous vein and laser treatment of superficial vein, and 189 lower limbs (accounting for $94 \%$ of the total number of lower limbs) were treated with local small point incision (length $\leq 1 \mathrm{~cm}$ ) for varicose vein exfoliation [9]. In order to avoid postoperative mobility inconvenience, only one side of the patient was operated each time. The operation interval on the other side was more than 1 month. A DIOMED 810nm laser (xxx company, England) was used with the pulse mode of the treatment for 1 second and the interval for 1 second in the operation. The power was $8 w-12 \mathrm{w}$ according to the position and degree of varicose vein. The incision (according to the patient's figure) was located $2 \mathrm{~cm}-3 \mathrm{~cm}$ below the groin, and the longitudinal line of the femoral artery pulsation was $2.5 \mathrm{~cm}$ to the inner side. The transverse incision was about $3 \mathrm{~cm}$ long. After detecting the great saphenous vein in the fat of the superficial fascial layer, the great saphenous vein was free and cut off. After the routine ligation of the proximal end, the suture was added. If the tributary was found, it was disconnected and ligated [10]. During the operation, a 0.035 inch super slide guide wire was placed retrogradely from the root of the great saphenous vein to the distal foot. When the guide wire was blocked, it could rotate or draw back and enter again until the advance stopped. Most of them could be seen that the guide wire was moving under the skin. After the position of the guide wire was determined, $5 \mathrm{~F}$ casing was inserted to the end of the guide wire, and then the guide wire was pulled out. When the laser guide wire end lights up, it was guided from the sleeve to the end of the sleeve, pulled out the sleeve $2 \mathrm{~cm}$, and fully exposed the heating part of the laser guide wire. The laser treatment was started by stepping on the pedal, the vein was closed with the heat at the end of the laser guide wire until the distal end of the great saphenous vein was ligated after pulling out the guide wire. Local varicose veins were guided into the guide wire by Trocar needle. The same method was used for laser treatment of varicose veins in other parts of the lower limbs. For the superficial vein with serious tortuous dilatation, the caliber larger than $8 \mathrm{~mm}$ or the nearest distance between the varicose part of great saphenous vein and the skin surface is less than $4 \mathrm{~mm}$, local stripping and extraction were carried out to avoid burning the skin. The patients were fol- 
lowed up for 1 month to 3 years.

\section{Results}

In this study, 214 cases of varicose veins of the lower extremities were treated by laser intracavity, 30 of them had complications or adverse events during or after the operation, accounting for $14 \%$ of cases. Data presented in Table 1 shows that during the operation, there were 7 cases without great saphenous vein in the groin incision, 3 cases with root injury of great saphenous vein, 3 cases with guide wire entering deep vein, 1 case with guide wire breaking, and 1 case with cannula burning through and breaking. Data presented in Table 2 shows that postoperatively8 cases of local skin and soft tissue thermal injury, 3 cases of saphenous nerve burn, 2 cases of lower extremity deep vein thrombosiswere found. No injury of femoral artery and foreign body remained during and after operation. Postoperative follow-up ranged from 1 month to 3 years. There were 2 cases of deep vein thrombosis. After treatment, PTS appeared. Among the 3 patients with saphenous nerve injury, 1 had skin sensory disturbance in the lower leg and 2 had hypoesthesia. Two patients with recurrent iliac vein stenosis were found by examination and cured by interventional operation.

Table 1. Intraoperative complications/adverse events

\begin{tabular}{cccc}
\hline Sl No. & Complications/adverse events & Number of cases & Probability of occurrence \% \\
\hline 1. & No great saphenous vein in groin incision & 7 & 3.2 \\
2. & Root injury of great saphenous vein & 3 & 1.4 \\
3. & Guide wire into deep vein & 3 & 1.4 \\
4. & Broken guide wire & 1 & 0.4 \\
5. & Casing burn through fracture & 1 & 0.4 \\
\hline
\end{tabular}

Table 2. Postoperative complications/adverse events

\begin{tabular}{cccc}
\hline SI No. & Complications/adverse events & Number of cases & Probability of occurrence \% \\
\hline 1. & Local skin and soft tissue thermal injury & 8 & 3.7 \\
2. & Burn of saphenous nerve & 3 & 1.4 \\
3. & Deep vein thrombosis of lower extremity & 2 & 0.9 \\
4. & Recurrence & 2 & 0.9 \\
\hline
\end{tabular}

\section{Discussion}

The treatment of great saphenous vein was not found during intraoperative exploration of groin incision. Although high ligation of great saphenous vein and intracavitary laser treatment have been widely used, there are still relevant risks and problems to be faced [11-12]. In case of improper treatment, severe consequences such as deep vascular injury and deep vein thrombosis may occur [13-14]. Among the 214 lower limb operations, 7 (3.2\%) had no great saphenous vein, which occurred in the obese patients. The reason could be related to tissue occlusion, anatomical variation and small diameter of saphenous vein root. After that, the skin was cut through the expanded great saphenous vein in the lower thigh, the medial knee joint or the upper leg, and the guide wire was introduced in the same direction as the blood flow. After calculating the length of the far and near incisions, the length of the guide wire into the great saphenous vein was controlled, and the slide of the guide wire was palpated at the end of the guide wire to the groin incision, so as to determine the exact location of the great saphenous vein in the groin incision. Then the great saphenous vein was ligated. If the guide wire was continued to move upward and enters the deep vein through the root of the great saphenous vein, the risk of deep vein thrombosis could increase. Therefore, itshould be avoided as far as possible. In addition, the intraoperative color Doppler ultrasound could be used to check the location of the great saphenous vein. It is easy to find the anatomical variation that has not been detected before the operation. However, the procedure was relatively time-consuming and may mistakenly treat other superficial veins as the great saphenous vein.

The failure to find the great saphenous vein in the incision increased the risk of ligation of the femoral vein which was mistakenly considered as the great saphenous vein, or at the same time damaged the femoral artery, leading to iatrogenic vascular injuries (IVI) and serious consequences, which were reported in literature [15-16]. It 
is very important to confirm the great saphenous vein during operation. If the operator has doubts, he canot clamp the damaged blood vessel with hemostatic forceps. The soft guide wire can be introduced into the puncture needle, and then the guide wire can be delivered to the distal end of the blood vessel. After the guide wire is palpated in the subcutaneous superficial vein, it can be confirmed that the guide wire is located in the superficial vein, and then the next operation can be carried out.

During the operation, when the root of the great saphenous vein was separated and ligated, the action should be gentle, and the operation should be carried out according to the characteristics of the anatomical structure. The rough or improper action could result in damage of saphenous vein. If the residual end of the great saphenous vein was too long, the possibility of secondary deep vein thrombosis would increase [16-18]. During the operation, the vein wall above the great saphenous vein root ligation should always be kept in a state of no tension or low tension, so as to avoid injury due to excessive traction. When the root of great saphenous vein was sutured, it was necessary to pay attention to the suture needle entering and ligating at one time, and did not repeatedly pierce the vein wall. In addition, vascular ligation should be firm and reliable to avoid loosening. All of these details may lead to deep vein thrombosis. If there was vascular injury at the root of the great saphenous vein during operation, anticoagulant treatment should be paid attention to prevent deep vein thrombosis. After operation, color ultrasound should be performed to confirm or exclude deep vein thrombosis as early as possible, so as to avoid delay in diagnosis and treatment [19].

The guide wire continued to enter the body, but the activity of the guide wire in the subcutaneous did not increase significantly. This kind of condition often occurs in the leg, if it was not detected during the operation, and the deep vein could be cauterized by laser, which may lead to deep vein thrombosis, with high risk [20-21]. Similar to this situation, the guide wire curls and gyrates in the varicose vein without serious consequences, but they are easy to let operator confused and to lead to entry into the deep vein without self-knowledge. In this case, after the guide wire enters the distal end of the great saphenous vein during the operation, it is necessary to closely observe the route of the guide wire under the skin, which produces light shadow contrast on the skin surface, which is easy to identify under the natural light, and the strong light of the surgical shadowless lamp is easy to cover up, so it is often necessary to turn off the surgical shadowless lamp for observation. Once the movement of the guide wire in the subcutaneous disappears suddenly, the guide wire should be pulled back immediately and then rotated to lead into the distal end. When the guide wire curls and convolutes in the superficial vein, it can be seen that the movement amplitude increases and the palpation vibration increases. Careful examination during the operation is not difficult to distinguish.

The breakage of guide wire or casing is rare, but the risk is high, which may lead to iatrogenic foreign body residue [22-23]. The reason of one case of guide wire fracture was that the guide wire was cut at the mouth of the trocar because the trocar needed to be further punctured but not pulled out when pulling out the guide wire. As the location of the superficial vein was found in time, the position of the guide wire of the broken part was determined by palpation, and then the broken guide wire was cut and taken out immediately. In this case, it should be noted to pull out the trocar before pulling the guide wire. If it is necessary to keep the trocar, it is necessary to follow the running direction of the guide wire, pull out the guide wire slowly, and pay attention to the resistance of the guide wire during the pulling process. Once there was resistance, the direction of the trocar should be adjusted until the resistance disappeared. The integrity of the guide wire shall be observed after the pulling out of the guide wire with resistance sense in the process of pulling out the guide wire, so as to discover the guide wire fracture in time and handle it to avoid [24]. The partial fracture of the casing was occurred with another case. Because the end of the laser guide wire was not completely penetrated through the casing, and it was blocked and stuck at the turning point of nearly $1 \mathrm{~cm}$ at the end of the casing. The laser guide wire was repeatedly heated and burned on the outside of the break for a long time, resulting in perforation on one side of the casing, but fortunately it was not completely broken and fell off. The reason of this kind of situation is that there is a $30^{\circ}$ bending design near the end of the casing, which is convenient for the operator to confirm that the laser guide wire goes through the casing. According to our experience, it is easy to be confused by the soft tissue barrier after the laser guide wire is penetrated. That is to say, there should be two blocking senses before and after the laser guide wire goes through the casing, the first is through the bend, and the second is the laser guide wire when goes through the casing and touches the vessel wall. It is necessary to pay attention to the sense of breakthrough and observe the red spot of laser. Compared with the anatomical position of the end of the sleeve, the red spot of laser light under the skin suddenly appears or the brightness suddenly increases, which means that the laser guide wire penetrates the sleeve and is exposed to the blood vessel. If there is any doubt, repeat the operation until it is confirmed that the laser guide wire has passed through the sleeve. 
Thermal injury of skin and subcutaneous tissue is hard to avoid because the superficial vein of the leg is adjacent to the cutaneous nerve [25-26]. When the laser guide wire is introduced into the local area for cauterization treatment, wet gauze block pressing is required to reduce the local skin temperature to prevent burns. Meanwhile, the vein is pressed to strengthen the effect of laser treatment. Because the laser guide wire head is covered, it is difficult to accurately determine the position. When the laser guide wire head has been pulled out from the subcutaneous to the skin surface, it is still hot and could burn skin. Most of them were point or cord like shallow $\mathrm{II}^{\circ}$ burns, occasionally with blisters. The injury was not serious, and could be disinfected and bandaged at the same time with the wound, with a good prognosis. During the operation, it was necessary to pay attention to the change of the position of the burn point, and at the same time, the wet yarn block was compressed when the superficial subcutaneous vein laser burns, which could reduce the damage degree when the laser guide wire head burned the skin by mistake.

\section{Conclusion}

Although laser treatment of varicose veins in the lower extremities has become a routine operation in many specialties, various specific conditions may still occur during the operation, and improper treatment may cause serious consequences. Only the principle and process of the operation have a more in-depth understanding and practical grasp. It is necessary to pay attention to observation, in order to avoid the occurrence of various complications and accidents. The minimally invasive advantage of this operation will be maximized.

\section{Acknowledgment}

We are grateful to the Principal \& MSVP of the People's Hospital, Shenzhen for providing permission and support to carry out this work. The authors also acknowledge the cooperation of the patients who participated in the study.

\section{References}

[1] Cao, G., Gu, H. C., Wang, J. T., et al. (2019). Comparison of endovenous laser treatment and high ligation in treatment of limb varicosity: A meta-analysis. Int Wound J., 2019 Jun; 16(3): 696-702. doi:10.1111/iwj.13083.

[2] Chehab, M., Dixit, P., Antypas, E., et al. (2015). Endovenous Laser Ablation of Perforating Veins: Feasibility, Safety, and Occlusion Rate Using a 1,470-nm Laser and Bare-Tip Fiber [J]. J Vasc Interv Radiol., 2015 Jun; 26(6): 871-7. doi: 10.1016/j.jvir.2015. 02.014.

[3] K. Rass, N. Frings, P. Glowacki, et al. Same Site Recurrence Is More Frequent After Endovenous Laser Ablation Compared With High Ligation and Stripping of the Great Saphenous Vein: 5 Year Results of a Randomized Clinical Trial (RELACS Study) [J]. Eur J Vasc Endovasc Surg, 2015 Nov: 50(5), 648-56. DOI: 10.1016/j.ejvs.2015.07.020.

[4] Arslan, N. Frings, P. Glowacki, et al. (2017). Same Site Recurrencth Less Energy in Endovenous Laser Ablation Treatment: Long-term Comparison of Bare-tip Fiber $980 \mathrm{~nm}$ Laser and Radial-tip Fiber $1470 \mathrm{~nm}$ Laser Application [J]. Ann Vasc Surg., 2017 Nov; 45: 166-172. doi: 10.1016/j.avsg.2017.06.042.

[5] Cavallini, A. (2015). Endovenous laser treatment of saphenous veins: is there clinical difference using different endovenous laser wavelenghts? Int Angiol [J]. 2015 Feb; 34(1): 1-8.

[6] Izzo, L., Pugliese, F., Pieretti, G., Izzo, S., et al. (2019). High ligation of sapheno-femoral junction and thermal ablation for lower limb primary varicosity in day hospital setting. Ann Ital Chir., 2019 Jun 28. pii: S0003469X19030264.

[7] K. Hartmann, D. Stenger, M. Hartmann, et al. (2017). Endovenous Ablation Versus Open Surgery for Varicose Veins: An Attempt at an Evaluation [J]. Hautarzt, 2017 Aug: 68(8), 603-613. DOI: 10.1007/s00105-017-3996-2.

[8] Knuth Rass, Norbert Frings, Paul Glowacki, et al. (2012). Comparable Effectiveness of Endovenous Laser Ablation and High Ligation With Stripping of the Great Saphenous Vein: Two-Year Results of a Randomized Clinical Trial (RELACS Study) [J]. Arch Dermatol, 2012 Jan: 148(1), 49-58. DOI: 10.1001/archdermatol.2011.272.

[9] Elrasheid A. H. Kheirelseid, Gillian Crowe, Rishabh Sehgal, et al. (2018). Systematic Review and Meta-Analysis of Randomized Controlled Trials Evaluating Long-Term Outcomes of Endovenous Management of Lower Extremity Varicose Veins [J]. J Vasc Surg Venous Lymphat Disord, 2018 Mar: 6(2), 256-270. DOI: 10.1016/j.jvsv.2017.10.012.

[10] Karsten Hartmann. (2020). Endovenous (Minimally Invasive) Procedures for Treatment of Varicose Veins: The Gentle and Effective Alternative to High Ligation and Stripping Operations [J]. Hautarzt, 2020 Jan: 71(1), 12-19. DOI: 10.1007/s00105-019-04520-2.

[11] Florio, G., Carnì, P., D'Amata, G., et al. (2019). Thermal ablation combined with high ligation of sapheno-femoral junction for lower limb primary varicosity [J]. G Chir., 2019 Sep-Oct; 40(5): 413-416.PMID: 32003720. 
[12] K. Rass, N. Frings, P. Glowacki, et al. (2015). Same Site Recurrence Is More Frequent After Endovenous Laser Ablation Compared With High Ligation and Stripping of the Great Saphenous Vein: 5 Year Results of a Randomized Clinical Trial (RELACS Study) [J]. Eur J Vasc Endovasc Surg., Nov 2015: 50 (5), 648-56. DOI: 10.1016/j.ejvs.2015.07.020.

[13] Alessandra Puggioni, Manju Kalra, Michele Carmo, et al. (2005). Endovenous Laser Therapy and Radiofrequency Ablation of the Great Saphenous Vein: Analysis of Early Efficacy and Complications [J]. J Vasc Surg., Sep 2005: 42(3), 488-93. DOI: 10.1016/j.jvs.2005.05.014.

[14] Cheng, Z. H., Wang, H. F., Chen, G., et al. (2010). Treatment of severe vascular injury caused by great saphenous vein varicose operation [J]. Chin J Gen Surg., 2010, 25(7): 523-525. DOI: 10.3760/cma.j.issn.1007-631X.2010.07.004.

[15] Robert Scott Brumberg. (2017). Cassie Davis. Ruptured Common Femoral Vein Pseudoaneurysm From a Common Femoral Arteriovenous Fistula Presenting as Lower Extremity Steal After Radiofrequency Ablation of the Great Saphenous Vein [J]. J Vasc Surg Venous Lymphat Disord., Jul 2017: 5(4), 571-574. DOI: 10.1016/j.jvsv.2016.12.016.

[16] Lu, S. Y., Qi, G. Y, Huang, X. T. (2006). Discuss for the complications of endovenous laser treatment of lower extremity varicose veins [J]. Chin J Gen Surg., 2006, 21(9): 657-659. DOI: 10.3760/j.issn: 1007-631X.2006.09.014.

[17] Khan, Y., Cheema, M. A., Abdullah, H. M. A., et al. (2019). Great saphenous vein stump: a risk factor for superficial/deep venous thrombosis and an indication for prophylactic anticoagulation?-a retrospective analysis [J]. Community Hosp Intern Med Perspect., 2019 Dec 14; 9(6): 473-476. doi: 10.1080/20009666.2019.1655626.

[18] Elizabeth Kudlaty, Babatunde Oriowo, Michael J. Yang, et al. (2017). Isolated Great Saphenous Vein Thrombus Is Associated With High Rates of Complications Regardless of Management [J]. Ann Vasc Surg., 2017 Nov: 45, 154-159. DOI: 10.1016/j.avsg.2017.05.028.

[19] I. Flessenkämper, M. Hartmann, K. Hartmann, et al. (2016). Endovenous Laser Ablation With and Without High Ligation Compared to High Ligation and Stripping for Treatment of Great Saphenous Varicose Veins: Results of a Multicentre Randomised Controlled Trial With Up to 6 Years Follow-Up [J]. Phlebology, 2016 Feb: 31(1), 23-33. DOI: $10.1177 / 0268355514555547$.

[20] Stefanie A. Gauw, James A. Lawson, Clarissa J. van Vlijmen-van Keulen, et al. (2016). Five-year Follow-Up of a Randomized, Controlled Trial Comparing Saphenofemoral Ligation and Stripping of the Great Saphenous Vein With Endovenous Laser Ablation (980 Nm) Using Local Tumescent Anesthesia [J]. J Vasc Surg., 2016 Feb: 63(2), 420-8. DOI: 10.1016/j.jvs.2015.08.084.

[21] Healy, D. A., Kimura, S., Power, D., et al. (2018). A Systematic Review and Meta-analysis of Thrombotic Events Following Endovenous Thermal Ablation of the Great Saphenous Vein [J]. Eur J Vasc Endovasc Surg., 2018 Sep; 56(3): 410-424. doi: 10.1016/j.ejvs.2018.05.008.

[22] Kichari, J. R., Salomonsz, R., Postema, R. R. (2008). Chronic pain due to a retained guidewire following endovascular laser therapy for varicose veins [J]. Ned Tijdschr Geneeskd., 2008 Jun 14; 152(24): 1387-90. PMID: 18664218.

[23] Öztürk, F., Atıc1, A., Asoğlu, R., et al. (2018). Percutaneous extraction of an intravascular guidewire retained for 1.5 years. Anatol J Cardiol., 2018 Dec; 20(6): E9. doi: 10.14744/Anatol J Cardiol.2018.98150.

[24] Lun, Y., Shen, S., Wu, X., et al. (2015). Laser fiber migration into the pelvic cavity: A rare complication of endovenous laser ablation [J]. Phlebology. 2015 Oct; 30(9): 641-3. doi: 10.1177/ 0268355514541982.

[25] G.-L. Jia, H.-L. Xi, X.-K. Wang, et al. (2018). Selective Retention of the Great Saphenous Vein to Prevent Saphenous Nerve Injury During Varicose Vein Surgery [J]. Eur Rev Med Pharmacol Sci., 2018(22), 3459-63. PMID: 25491621.

[26] Lynch, N. P., Clarke, M., Fulton, G. J. (2015). Surgical management of great saphenous vein varicose veins: A meta-analysis. Vascular [J]. 2015 Jun; 23(3): 285-96. doi: 10.1177/1708538114542633. 